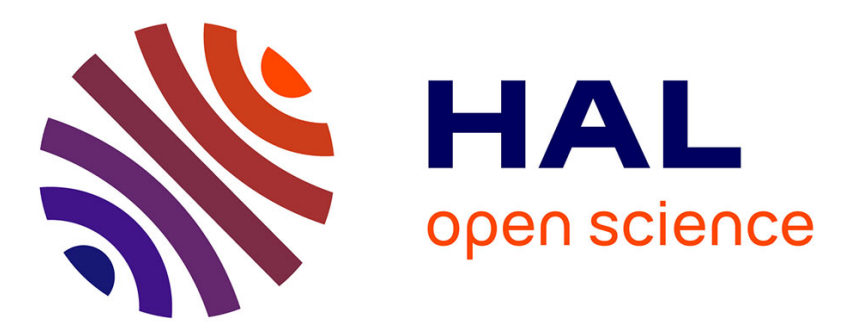

\title{
Integral Equation Study of the Square-Well Fluid for Varying Attraction Range
}

El Bahloul El Mendoub, Jean-Francois Wax, Isabelle Charpentier, Noel Jakse

\section{To cite this version:}

El Bahloul El Mendoub, Jean-Francois Wax, Isabelle Charpentier, Noel Jakse. Integral Equation Study of the Square-Well Fluid for Varying Attraction Range. Molecular Physics, 2009, 106 (24), pp.2667-2675. 10.1080/00268970802603499 . hal-00513237

\section{HAL Id: hal-00513237 \\ https://hal.science/hal-00513237}

Submitted on 1 Sep 2010

HAL is a multi-disciplinary open access archive for the deposit and dissemination of scientific research documents, whether they are published or not. The documents may come from teaching and research institutions in France or abroad, or from public or private research centers.
L'archive ouverte pluridisciplinaire HAL, est destinée au dépôt et à la diffusion de documents scientifiques de niveau recherche, publiés ou non, émanant des établissements d'enseignement et de recherche français ou étrangers, des laboratoires publics ou privés. 


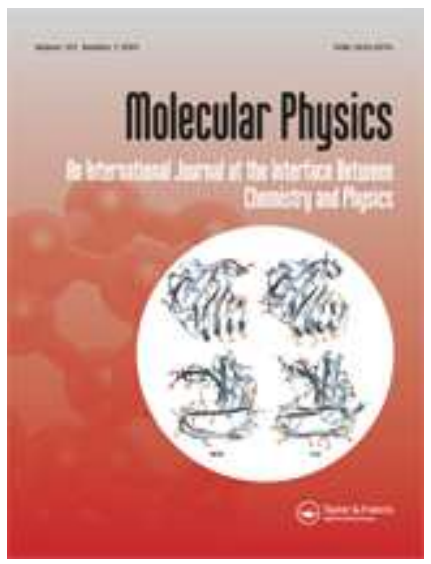

\section{Integral Equation Study of the Square-Well Fluid for Varying Attraction Range}

\begin{tabular}{|r|l|}
\hline Journal: & Molecular Physics \\
\hline Manuscript ID: & TMPH-2008-0209.R1 \\
\hline Manuscript Type: & Full Paper \\
\hline Date Submitted by the & 28-Oct-2008 \\
\hline Complete List of Authors: & $\begin{array}{l}\text { El Mendoub, El Bahloul; Université Paul Verlaine Metz, LPMD } \\
\text { Wax, Jean-Francois; Université Paul Verlaine Metz, LPMD } \\
\text { Charpentier, Isabelle; Université Paul Verlaine Metz, LPMM } \\
\text { Jakse, Noel; Grenoble Institute of Technology, SIMAP }\end{array}$ \\
\hline Keywords: & $\begin{array}{l}\text { Integral equations, square-well fluid, liquid-vapour coexistence, } \\
\text { colloids }\end{array}$ \\
\hline $\begin{array}{l}\text { Note: The following files were submitted by the author for peer review, but cannot be converted } \\
\text { to PDF. You must view these files (e.g. movies) online. }\end{array}$ \\
\hline manuscript-revised.tex
\end{tabular}

\section{scholarONE" Manuscript Central}




\title{
Integral Equation Study of the Square-Well Fluid for Varying Attraction Range
}

\author{
E. B. El Mendoub ${ }^{1}$, J.- F. Wax ${ }^{1}$, I. Charpentier ${ }^{2}$ and N. Jakse ${ }^{3}$ \\ ${ }^{1}$ Laboratoire de Physique de la Matière Dense, \\ Université Paul Verlaine Metz, 1, bd F. D. Arago, \\ CP 87811, 57078 Metz Cedex 3, France \\ ${ }^{2}$ Laboratoire de Physique et Mécanique des Matériaux, \\ UMR CNRS 7554, Ile du Saulcy, 57045 Metz Cedex 1, France and \\ ${ }^{3}$ Laboratoire Sciences et Ingénierie, Matériaux et Procédés (SIMAP), \\ UMR CNRS 5266, PHELMA- Grenoble INP, \\ BP 75, 38402 Saint Martin d'Hères Cedex, France.
}

\begin{abstract}
In the present work, the properties of the square-well fluid (SW) for varying attraction range are investigated in the framework of the integral equation method. The pair-correlation functions are calculated for a wide range of the potential attraction including the colloidal regime, and the liquid-vapour phase diagram as well as the critical parameters are determined for attraction ranges spanning from the van der Waals limit down to the metastable region. An overall good agreement is found compared to available simulation data as well as several theoretical approaches.
\end{abstract}




\section{INTRODUCTION}

Complex systems such as colloidal suspensions or protein solutions have attracted much attention from the scientific community. An important issue in this field is to understand the role played by the range of attraction in controlling of the thermodynamics and the phase behavior in such systems driven by complex effective interactions ${ }^{1}$. It has been argued that the details of the interactions might have little influence, and a generalized law of corresponding states seems to govern the system. The size of the particles mapped by an equivalent hard-sphere (HS) diameter and the second virial coefficient, containing a simple energetic parameter, act as the scaling variables $^{2-4}$. This reinforces the possibility to describe such kind of systems with very simplified models of interactions.

Interestingly, the square-well (SW) potential whose the potential energy as a function of the distance of separation $r$ reads

$$
u(r)=\left\{\begin{array}{cc}
\infty, & r \leq \sigma, \\
-\varepsilon, & \sigma<r \leq \lambda \sigma, \\
0 & r>\lambda \sigma,
\end{array}\right.
$$

which is the simplest model that exhibits a liquid-vapour equilibrium and obeys a law of corresponding states. Its three parameters are the size of the hard spheres, $\sigma$, the strength $\varepsilon$, and the width $\lambda$ of the potential-well. Obviously, the HS potential is recovered when $\varepsilon=0$, otherwise the range of attraction can be controlled by varying $\lambda$ such that various kind of fluids can be treated. The infinitely short-range limit $(\lambda \rightarrow 1$, and $\varepsilon \rightarrow \infty)$ corresponds to the sticky HS model ${ }^{5}$ and short ranges of the potential $(\lambda<1.25)$ are taken as models of colloidal suspensions ${ }^{6}$ and protein solutions ${ }^{7}$. Intermediate ranges $(1.5 \leq \lambda<2)$ intend to mimic simple fluids, and longer ranges correspond to very shallow potentials, for instance with $\lambda \rightarrow \infty$, corresponding to the van der Waals mean field theory, a limit which is numerically reached ${ }^{8}$ for $\lambda=3$. Therefore, due to the simplicity of its mathematical expression and its applicability to a wide class of fluids, the SW model represents a useful benchmark for theoretical developments. Most of the works reported in the literature were devoted to the intermediate-ranged potentials for which the model behaves in a qualitative manner as a normal fluid. Some interest has regained recently since it has been shown that short ranges are particularly well-suited to the description of colloids ${ }^{6,9,10}$.

A large amount of work was devoted to the understanding of the structural and thermodynamic properties including the liquid-vapour phase coexistence of the SW fluids. Numerical simulations, based on molecular dynamics and Monte-Carlo (MC) methods ${ }^{8,11-15}$, were mostly used to study 
the SW potential for the intermediate-ranges of the potential. Only a few of them were devoted to the short-ranged domain ${ }^{2,15,16}$. Analytical theories for the structure ${ }^{17,18}$, thermodynamics ${ }^{19}$, and liquid-vapour coexistence ${ }^{20}$ were developed on the basis of either the van der Waals theory or HS perturbation method or the renormalization group (RG) approach ${ }^{21}$ for various ranges of the potential. Besides, semi-analytical methods, based on the statistical physics of fluids such as the integral equations (IE), which consists in solving numerically the Ornstein-Zernike (OZ) equation together with an approximate closure relation have been thoroughly used in the seventies and eighties $^{22}$. In this framework, the hierarchical reference theory (HRT) $)^{23}$ and the self-consistent OZ approximation (SCOZA) ${ }^{24}$ are successful to deal with the critical behaviour of the SW fluids for various ranges of attraction.

Of particular interest is the integral equation proposed by Sarkisov ${ }^{25}$ to treat the structure and the thermodynamics of fluids, which has proven to achieve a good degree of thermodynamic consistency $^{26}$. It has been previously applied to a wide class of Lennard-Jones type potentials ${ }^{27}$ and also to discontinuous potentials such as the $\mathrm{HS}^{28}$ and the attractive Yukawa potentials ${ }^{29}$. The aim of the present work is to investigate the properties of the SW fluids covering attraction part of the potential from the short-range to the van der Waals limit on the same footing. For this purpose, the have carried out calculations using the Sarkisov ${ }^{25,29}$ integral equation method coupled with efficient numerical tools, namely, the tangent linear method to get exact derivatives and an adaptative technique ${ }^{27}$. We show that within the present IE approach: (i) a correct description of the pair-correlation functions can be obtained for a wide range of the potential attraction including the colloidal regime and (ii) the liquid-vapour phase diagram as well as the critical parameters can be calculated for the whole domain of $\lambda$ for which coexistence exists.

The layout of the paper is the following. Section 2 briefly describes the integral equation method, the thermodynamic properties as applied to the SW model, as well as the numerical techniques to construct the liquid-vapour phase diagrams. The results for the structural properties and the liquid vapour coexistence are presented and discussed in Section 3. In Section 4 we summarize and conclude. 


\section{THEORETICAL BACKGROUND}

\section{A. Integral equations}

Let us consider a fluid at a temperature $T$, and number density $\rho$ in which the particles interact through a pair-potential $u(r)$. According to the OZ equation, the total correlation function, $h(r)$, between two particles is defined as the sum of the direct correlation function, $c(r)$, and the indirect correlation function, $\gamma(r)$ :

$$
h(r)=c(r)+\rho \int h\left(r^{\prime}\right) c\left(\left|\mathbf{r}-\mathbf{r}^{\prime}\right|\right) d \mathbf{r}^{\prime}=c(r)+\gamma(r) .
$$

The integral equation method consists in solving Eq. (2) together with a closure relation whose formal expression for the pair-correlation function $g(r)(=h(r)+1)$ is

$$
g(r)=\exp [-\beta u(r)] \exp [\gamma(r)+B(r)]
$$

$\beta=1 / k_{B} T$ being the inverse temperature and $k_{B}$ Boltzmann's constant. The bottleneck of the method is that Eq. (3) depends on the bridge function, $B(r)$, which represents an infinite sum of elementary bridge diagrams ${ }^{30}$. Following Sarkisov's scheme ${ }^{25}$ the bridge function is written as

$$
B(r)=\left[1+2 \gamma^{*}(r)\right]^{1 / 2}-1-\gamma^{*}(r) .
$$

The function $B(r)$ is a functional in terms of the indirect correlation function and also of the interaction potential. In this closure relation, a renormalization concept of the indirect correlation function has been introduced by considering $\gamma^{*}(r)$ rather than $\gamma(r)$ in $B(r)$. Such an idea was implemented by Lee et $a ._{.}^{32}$ for the Lennard-Jones with $\gamma^{*}(r)=\gamma(r)-\beta u_{2}(r)$, where $u_{2}(r)$ is the attractive part of the pair potential according to the Weeks, Chandler and Andersen (WCA) splitting $^{31}$. For the hard-sphere (HS) potential, an efficient renormalization was found, namely $\gamma^{*}(r)=\gamma(r)+\rho f(r) / 2, f(r)(=\exp (-\beta u(r))-1)$ being the Mayer function ${ }^{33}$. In a recent work ${ }^{29}$, we have extended this renormalization to the hard-core Yukawa potential as

$$
\gamma^{*}(r)= \begin{cases}\gamma(r)+\rho f\left(\sigma^{+}\right) / 2, & r<\sigma, \\ \gamma(r)+\rho f(r) / 2, & r \geq \sigma\end{cases}
$$

that we adopt also here for the SW model of interaction. The renormalized indirect correlation function is then a discontinuous function. However this poses no conceptual problem since the numerical solution is obtained through the LVM method in the Fourier space where the correlation functions are continuous. 
The compressibility factor $Z$ is determined from the virial equation of states such as :

$$
Z=\frac{\beta P}{\rho}=1+\frac{2 \pi \rho \sigma^{3}}{3}\left\{g\left(\sigma^{+}\right)-\lambda^{3}\left[g\left((\lambda \sigma)^{-}\right)+g\left((\lambda \sigma)^{+}\right)\right]\right\},
$$

where $P$ is the pressure. The inverse isothermal compressibility, $1 / \chi_{T}$, is obtained from a density derivative of the pressure

$$
\begin{aligned}
\beta \frac{\partial P}{\partial \rho} & =1+\frac{4 \pi \rho \sigma^{3}}{3 \beta} g\left(\sigma^{+}\right)+\frac{2 \pi\left(\rho \sigma^{3}\right)^{2}}{3 \beta} \frac{\partial g\left(\sigma^{+}\right)}{\partial \rho} \\
& -\frac{4 \pi \rho(\sigma \lambda)^{3}}{3}\left\{\left[g\left((\sigma \lambda)^{+}\right)-g\left((\sigma \lambda)^{-}\right)\right]+\frac{\rho}{2}\left[\frac{\partial g\left((\sigma \lambda)^{+}\right)}{\partial \rho}-\frac{\partial g\left((\sigma \lambda)^{-}\right)}{\partial \rho}\right]\right\}
\end{aligned}
$$

Finally, the excess chemical potential is calculated using the approximate form proposed by Kyseliov and Martynov ${ }^{34}$

$$
\beta \mu^{\mathrm{ex}}=4 \pi \rho \int_{0}^{\infty}\left[\gamma^{*}(r)+B(r)-h(r)+\frac{1}{2} h(r)\left\{\gamma^{*}(r)+\frac{4}{3} B(r)\right\}\right] r^{2} d r .
$$

The construction of the liquid-vapour coexistence curves is based on conditions of thermal, mechanical and chemical equilibria. The thermodynamic points of the liquid $(l)$ and vapour $(v)$ phases belonging to the binodal line satisfy the relations:

$$
T_{l}=T_{v}, \quad P_{l}=P_{v}, \quad \mu_{l}=\mu_{v} .
$$

The chemical potentials $\mu_{l}$ and $\mu_{v}$ are obtained from Eq.(9) by $\mu=\mu^{\mathrm{ex}}+k_{\mathrm{B}} T \ln \left(\rho \sigma^{3}\right)$.

The domain of mechanical stability is limited by the spinodal line, which can be obtained from the state points corresponding to the extrema of the virial pressure along each isotherm. These are the points in the metastable region where the inverse isothermal compressibility given by Eq. (8) becomes zero. As argued by Sarkisov ${ }^{26}$, when approaching the instable region, correlation functions including $g(r)$ itself display a diverging correlation radius that affects the thermodynamic calculations due to long-range truncation effects. However, the inverse compressibility calculated from the virial route and given by Eq. (8) does not depend explicitely on the asymptotic form of the correlation functions, as only the values of $g(r)$ at the discontinuities are involved, and therefore 
it is not affected by these long-range effects. Consequently, the right-hand side of Eq. (8) can be calculated as soon as a valid solution of the IE exists, as it is not subject to long range truncation effects when approaching the unstable region. We thus define the spinodal line ${ }^{35}$ as a zero value of Eq. (8). Our experience is that the quantity $1 / \chi_{T}$ can be negative when a solution of IE is found in the instable region being therefore beyond the spinodal line.

\section{Numerical techniques}

The accurate calculation of the structure, thermodynamics and the liquid-vapour coexistence curve relies on efficient numerical tools that are presented briefly in this paragraph.

First, a numerical solution of the pair-correlation function within the integral equation is obtained through the algorithm of Labik et al. ${ }^{36}$. It combines the Newton-Raphson and the successive substitution (standard Picard scheme) methods guaranteeing a good stability and a fast solution. In a first stage, successive approximations of the indirect correlation function $\gamma(r, \rho, T)$ for a given state point $(\rho, T)$ are generated through the following iterative process:

$$
\gamma_{i+1}(r, \rho, T)=\mathcal{M}\left[\gamma_{i}(r, \rho, T)\right]
$$

where $\mathcal{M}$ is a nonlinear functional derived from the $\mathrm{OZ}$ equation in the reciprocal space as

$$
\tilde{\gamma}(q)=\frac{\rho \tilde{c}^{2}(q)}{1-\rho \tilde{c}(q)} .
$$

The direct correlation function $\tilde{c}(q)$ is expressed as a function of $\gamma(r)$ in the direct space by means of the closure relation, which in the present work is given by Eqs. (4) and (5). The second stage corresponds to the Newton-Raphson part, in which the functional $\mathcal{M}$ is linearized with respect to $\gamma$ as:

$$
\mathcal{M}[\gamma] \simeq \mathcal{M}\left[\gamma_{0}\right]+\Delta \gamma \cdot \frac{\partial \mathcal{M}}{\partial \gamma}\left[\gamma_{0}\right]
$$

about initial guess $\gamma_{0}(r)$ assumed to be sufficiently close to the solution $\gamma(r)$, i.e. $\gamma(r)=\gamma_{0}(r)+$ $\Delta \gamma(r)$ with $\Delta \gamma(r)$ small (the interested reader is referred to ${ }^{36}$ for the specific expression of $\mathcal{M}$ as well as technical details). The NR method, which consists in estimating the unknown difference $\Delta \gamma$ at each step of the iterative process given by Eq. (11) converges quadratically. Finally, a solution at state point $(\rho, T)$ is found when $\Delta \gamma$ vanishes. From a technical point of view, the correlation functions are discretized in the $r$ space on a grid of 4096 points with a mesh of $0.0125 \sigma$, which implies a spatial extension $R_{\max }=51.2 \sigma$, sufficient for an accurate numerical integration and a fast Fourier transform. 
Secondly, the determination of the thermodynamic quantities of interest have expressions containing not only the pair-correlation function $g(r)$ but also its thermodynamic derivatives either with respect to density or temperature that have to be evaluated. This is the case for the isothermal compressibility given by Eq. (8). For this purpose the tangent linear method ${ }^{37}$ gives us the possibility to compute locally all the thermodynamic functions in an "exact" manner for each state point $(T, \rho)$, i.e. without having recourse to a finite difference method. The tangent linear technique is applied directly to the code of Labik et al., using an automatic differentiation tool, namely, the software ODYSSEE ${ }^{39}$ (we refer the interested reader to previous papers for a more detailed description ${ }^{40}$ ).

Finally, as the thermodynamic properties are calculated locally at any state point $(T, \rho)$, an adaptative technique enables us to perform an efficient and accurate systematic mapping of the thermodynamic space, in order to construct easily the binodal and spinodal lines as close to the critical point as possible. The detailed description can be found in a reference $\operatorname{paper}^{27}$, and will not be repeated here. It is worth emphasizing that the adaptative technique used here drives the whole process and makes calls to the differentiated code of Labik et al. at each state point needed for the mapping. The latter is started with an initial guess $\gamma_{0}(r, \rho, T)$ and the solution is computed at the nearby state point $(\rho, T+\Delta T)$ where $\Delta T$ is small enough to get a small difference $\Delta \gamma$, which is one of the keys for a fast resolution of the Labik et al. algorithm for successive state points. Technically, a good compromise between accuracy and speed for the two-dimensional grid is to use an initial temperature step $\Delta T=0.01 k_{\mathrm{B}} / \varepsilon$ and density step $\Delta \rho=0.01 \sigma^{-3}$.

\section{RESULTS AND DISCUSSION}

The central quantities entering the calculation of the thermodynamic functions needed to construct the liquid-vapour phase diagram are the pair-correlation function $g(r)$ and its temperature and density derivatives. A comparison with simulation data offers the possibility of probing the IE directly at the microscopic level. In Figures 1 and 2, the calculated $g(r)$ are respectively plotted for values of $\lambda$ in the domain [1.3;2.0], belonging to the intermediate attraction range, and $[1.05 ; 1.2]$ corresponding to the colloidal regime. Our results are compared with existing Monte-Carlo simulations in the canonical ensemble $(N V T)$ by Largo et al. ${ }^{18}$. In the intermediate attraction range, the curves reveal an excellent agreement with the MC simulation data at both low and high densities, whatever the values of $\lambda$. The height and shape of the first peak is well reproduced, as well as the subsequent oscillations for $r>\lambda$. In the short range of attraction range, the curves of Figure 
2 show a reasonable agreement at low density. At high densities, the IE method underestimates substantially the MC simulation data regarding the height of the first peak of $g(r)$ and overestimates the oscillations for distances just beyond $r=\lambda$. It is worth mentioning however that the concordance is still reasonable for $\lambda=1.2$.

As can be seen in Eqs. (6) to (8), the values of $g\left(\sigma^{+}\right), g\left(\lambda^{-}\right)$and $g\left(\lambda^{+}\right)$drive the thermodynamics of the SW fluid. Table I gathers these values as well as the corresponding MC data of Largo et al. ${ }^{18}$ to provide a general and quantitative view of the performance of the present IE scheme. Different values of $\lambda$ are considered in the short and intermediate attraction range, as a function of density for several isotherms. The general trends that emerge from the analysis of Table I confirms the one inferred from the curves of $g(r)$. The results are especially in good accordance for the intermediate range of $\lambda$. They remain good with the simulation data in the domain of $\lambda$ corresponding to the short range of attraction at low and moderate densities, provided that the temperature is not too low. The disagreement with the simulation data limits to $\lambda=1.05$ and $\lambda=1.1$ at high density, as already seen for the pair-correlation function. Finally, the results found in Table I demonstrate the reliability of the present IE scheme, i.e. its ability to describe the structural properties of the SW fluid over a wide domain of thermodynamic states as well as of interaction ranges $\lambda$, except for the two lowest values of $\lambda$.

A stringent test for the reliability of the present integral equation approach is to determine the liquid-vapour phase diagram and to compare the predicted results to available simulation data. We recall that the accuracy of the phase diagram lies on the quality of the calculated thermodynamic functions which in turn depend essentially on the values of $g\left(\sigma^{+}\right), g\left(\lambda^{-}\right)$and $g\left(\lambda^{+}\right)$studied above, as well as their thermodynamic derivatives calculated in an exact manner through tangent linear technique. On the one hand, the pressure and the chemical potential given respectively by Eqs. (7) and (9) are needed for the binodal line and, on the other hand, the inverse compressibility (Eq. (8)), that controls the mechanical stability of the system, yields the spinodal line. For the determination of the critical parameters, we follow the technique described in our preceeding work ${ }^{27}$ and we refer the reader to it for further details. In this method, the error bars are controled by fixing the minimum value of the temperature step and that of the density step of the adaptative grid. In the vicinity of the critical point these are $0.001 k_{\mathrm{B}} / \varepsilon$ and $0.001 \sigma^{-3}$, respectively.

Let us first examine the results for the long attraction range $(2<\lambda \leq 3)$. To our best knowledge, no simulation data exist for $\lambda>3$, above the van der Waals limit ${ }^{8}$. Figure 3 shows the IE curves which are compared to those of the SCOZA method as well as to the Gibbs ensemble Monte-Carlo (GEMC) simulation data of Patel et al. ${ }^{13}$. For $\lambda=3$ (upper curve) the GEMC simulation data of 
Orkoulas and Panagiotopoulos ${ }^{12}$ as well as the molecular dynamics results of Benavides et al. ${ }^{8}$ have also been included. For the four values of $\lambda$ covering this range, the IE method yields a binodal line in very good agreement with the simulation data. It appears that the IE and SCOZA ${ }^{24}$ predictions are of comparable accuracy for the high density branch, while the SCOZA seem to be slightly better for the low density branch for $\lambda=2.8$ and 3. The critical parameters are gathered in Table II along with the simulation data when available and with those of theoretical approaches, namely SCOZA, $\mathrm{HRT}^{23}$ and $\mathrm{RG}^{21}$. These have been inferred within the IE scheme from the position of the maximum of the spinodal line. The obtained values are close to SCOZA, HRT and RG ones, especially, the critical temperature.

Figure 4 shows the liquid-vapour phase diagram for the intermediate attraction range $(1.5 \leq$ $\lambda \leq 2)$. An overall good agreement is found with respect to GEMC data of Patel et al. ${ }^{13}$ for the three values of $\lambda$ considered. Interesting enough, for the high density (liquid) branch, it appears that the IE gives better results than the SCOZA for $\lambda=1.5$ and $\lambda=1.75$. For the coexistence curves, calculated for the shorter range of attraction and shown in Figure 5, a different situation emerges. In the case of $\lambda=1.25$, both the IE and SCOZA, which give similar results, fail to reproduce the high density branch when compared to the simulation data of Patel et al. ${ }^{13}$ and del Rio et $a{ }^{14}{ }^{14}$. For $\lambda=1.375$, this is also the case, nevertheless the IE approach gives better predictions. It is worth mentioning however that, for $1.25 \leq \lambda \leq 2$ the critical parameters inferred from the IE (see Table II) are close to the SCOZA ones, and are also in reasonable agreement with the MC data. It appears that, away from the critical region, the IE is more reliable than the SCOZA for the liquid branch of the coexistence curve.

As can be seen on Figures 3, 4, and 5, the temperature range of the liquid-vapour coexistence is reduced with decreasing values of $\lambda$. The question of the disappearance of the liquid-vapour coexistence with the narrowing of the potential well has been studied for various type of potentials ${ }^{41}$. For the SW potential, it is worth mentioning that Tejero and co-workers ${ }^{42}$ have shown that the simple van der Waals theory predicts a vanishing of the coexistence around $\lambda=1.25$. More recently, Liu et al. ${ }^{16}$ using GEMC simulation in conjunction with an aggregation volume biased technique have pointed out that the triple point disappears for $\lambda=1.25$ and a sublimation line takes place for all temperatures. The liquid-vapour coexistence, which can nevertheless be constructed, become metastable and hidden under it. Such findings could explain the difficulties encountered by both the IE and SCOZA methods to reproduce the binodal line for this value of $\lambda$. Liu et al. have shown that such a "metastable" liquid-vapour coexistence could be determined by simulation ${ }^{16}$ down to $\lambda=1.15$. In the same spirit, we have tested the ability of the IE method to determine 
the liquid-vapour coexistence curves for values of $\lambda$ below 1.25, and Figure 5 shows the binodal lines obtained for $\lambda=1.20$ and $\lambda=1.175$. The critical temperatures and densities predicted by our IE approach are lower that those obtained from HRT. We mention that below $\lambda=1.175$, no binodal line could be calculated and the value of 1.175 can by considered as the limit for the present integral equation scheme, which is very close to that found from the computer simulation ${ }^{16}$.

\section{CONCLUSION}

To summarize the integral equation method is used to study the properties of the squarewell fluid for varying attraction range. The calculations are carried out with the self-consistent closure relation proposed by Sarkisov ${ }^{25}$. We have shown that the pair-correlation functions can be accurately described for a wide range of the potential attraction including the colloidal regime by comparison with simulation data. For the smallest values of $\lambda$ (1.05 and 1.1) correct results are obtained only at low densities and high temperatures.

The liquid-vapour phase diagram as well as the critical parameters are obtained for $1.175 \leq$ $\lambda \leq 3$, using the adaptative technique associated to the tangent linear method to get exact thermodynamic derivatives of the pair-correlation function. In particular, for long attraction range $(2<\lambda \leq 3)$ the integral equations predicts accurately the binodal line with respect to the simulation data. Our results are close to those obtained from the SCOZA method ${ }^{24}$, including the critical parameters. In the intermediate range of attraction $(1.5 \leq \lambda \leq 2)$, a reliable description of the liquid-vapour coexistence curves and critical parameters is found, while for shorter values $(1.25 \leq \lambda<1.5)$, namely down to the stability limit ${ }^{16}$ the results are only in qualitative agreement with the simulation data, especially for the liquid branch of the binodal. Nevertheless, it appears that for the intermediate and short ranges, the latter is generally better described by the IE scheme than the SCOZA. Results for the liquid-vapour phase diagram is also obtained in the region with $\lambda<1.25$, and a limit of $\lambda=1.175$ is found, close to that obtained by state-of the-art simulation technique ${ }^{16}$.

1 M. H. J. Hagen, E. J. Meijer, G. C. A. M. Mooij, D. Frenkel, and H. N. W. Lekkerkerker, Nature (London) 365, 425 (1993).

2 M. Noro and D. Frenkel, J. Chem. Phys. 113, 2941 (2000).

3 G. Foffi, C. D. Michele, F. Sciortino, and P. Tartaglia, Phys. Rev. Lett. 94, 078301 (2005). 
4 G. Foffi, and F. Sciortino, Phys. Rev. E 74, 050401(R) (2006).

5 J. Largo, M. A. Miller, and F. Sciortino, J. Chem. Phys. 128, 134513 (2008).

${ }^{6}$ K. Dawson, G. Foffi, M. Fuchs, W. Götze, F. Sciortino, M. Sperl, P. Tartaglia, Th. Voigtmann, and E. Zaccarelli, Phys. Rev. E 63, 011401 (2001); G. Foffi, G. D. McCullagh, A. Lawlor, E. Zaccarelli, K. Dawson, F., Sciortino, P. Tartaglia, D. Pini, and G. Stell, Phys. Rev. E 65, 031407 (2002).

7 A. Lomakin, N. Asherie and G. B. Bendek, J. Chem. Phys. 104, 1646 (1996);.J. F. Lutsko, and G. Nicolis, J. Chem. Phys. 122, 244907 (2005).

8 A. L. Benavides, F. del Río, and J. Alejandre, Mol. Phys. 74, 321 (1991).

9 A. Lang, G. Kahl, C. N. Likos, H. Löwen, and M. Watzlawek, 1999, J. Phys.: condens. Matter 11,10143 (1999);

10 E. Zaccarelli, G. Foffi, K. A. Dawson, S. V. Buldyrev, F. Sciortino, and P. Tartaglia, J. Phys.: condens. Matter 15, S367 (2003).

11 P. Bolhuis, M. Hagen, and D. Frenkel, Phys. Rev. E 50, 4840(1994).

12 G. Orkoulas and A. Z. Panagiotopoulos, J. Chem. Phys. 110, 1581 (1999).

13 B. H. Patel, H. Docherty, S. Varga, A. Galindo, and G. C. Maitland, Mol. Phys. 103, 129 (2005).

14 F. del Rio, E. Avalos, R. Espindola, L. F. Rull, G. Jackson, and S. Lago. Mol. Phys. 100, 2531 (2002).

15 R. Lopez-Rendon, Y. Reyes, and P. Orea, J. Chem. Phys. 125, 084508 (2006).

16 H. Liu, S. Garde, and S. Kumar, J. Chem. Phys. 123, 174505 (2005).

17 J. Largo and J. R. Solana, Fluid Phase Equilib. 193, 277 (2002).

18 J. Largo, J. R. Solana, S. B. Yuste and A. Santos, J. Chem. Phys. 122, 084510 (2005).

19 J. Largo, and J. M. Solana, Phys. Rev. E 67, 066112 (2003).

20 A. L. Benavides and F. del Rio, Mol. Phys. 68, 983 (1989); A. Gil-Villegas, F. del Rio, and A. L. Benavides, Fluid Phase Equilib. 119, 97 (1996).

21 J. A. White, J. Chem. Phys. 113, 1580 (2000).

22 C. Caccamo, Phys. Rep. 274, 1(1996).

23 A. Reiner, G. Kahl, J. Chem. Phys. 117, 4925 (2002).

24 E. Scholl-Paschinger, A. L. Benavisdes, and R. Castañeda-Priego, J. Chem. Phys. 123, 234513 (2005).

25 G. Sarkisov, J. Chem. Phys., 114, 9496 (2001).

26 G. Sarkisov, J. Chem. Phys. 119, 373 (2003).

27 I. Charpentier and N. Jakse, J. Chem. Phys. 123, 204910 (2005).

28 E. B. E. Mendoub, Thèse de Doctorat (Université Paul Verlaine-Metz 2008).

29 E. B. El Mendoub, J.-F. Wax, and N. Jakse, Phys. Rev. E 74, 052501 (2006).

30 G. A. Martynov, Fundamental Theory of Fluids. Methods of Distribution Functions (Higer, Bristol 1992).

31 J. D. Weeks, D. Chandler, and H. C. Andersen, J. Chem. Phys. 54, 5237 (1971).

32 L. L. Lee, D. Ghonasgi and E. Lomba, J. Chem. Phys. 104, 8058 (1996).

33 L. L. Lee, J. Chem. Phys. 103, 9388 (1995); ibid., J. Chem. Phys. 107, 7360 (1997).

34 O. E. Kiselyov, and G. A. Martynov, J. Chem. Phys. 93, 1942 (1990). 
35 P.G. Ferreira, R. L. Carvalho, M. M. Telo da Gama and A. G. Schlijper, J. Chem. Phys. 101, 594 (1985).

36 S. Labik, A. Malijevski, and P. Vonka, Mol. Phys. 56, 709 (1985).

37 A. Griewank and C. F. Corliss (Eds.), Automatic Differentiation of Algorithms: Theory, Implementation and Applications (SIAM, Philadelphia 1991).

38 A. Griewank, Evaluating Derivatives: Principles and Techniques of Algorithmic Differentiation, Frontiers in Applied Mathematics (SIAM 2000).

39 C. Faure and Y. Papegay, Odyssée Version 1.6: The language reference manual, Rapport Technique INRIA RT-211 (1997). The interested reader is referred to the Internet site autodiff.com for a review of available automatic differentiation tools.

40 I. Charpentier and N. Jakse, J. Chem. Phys. 114, 2284 (2001); N. Jakse, and I. Charpentier, Phys. Rev. E 67, 061203 (2003).

41 G. Malescio, J. Phys.: Condens. Matter 19, 073101 (2007).

42 C. F. Tejero, A. Dannoun, H. N. V. Lekkerkerker, and M. Baus, Phys. Rev. Lett. 73, 752 (1994); A. Dannoun, C. F. Tejero, and M. Baus, Phys. Rev. E 50, 2913 (1994). 
Figure 1: Pair-correlation function $g(r)$ in the intermediate attraction range for density $\rho \sigma^{3}=$ 0.2 (a), and $\rho \sigma^{3}=0.8$ (b). In both panels, the solid, dashed and dotted lines correspond respectively to the IE results for $\lambda=1.30$ and $k_{B} T / \varepsilon=1.0, \lambda=1.50$ and $k_{B} T / \varepsilon=1.0$, and $\lambda=2.0$, and $k_{B} T / \varepsilon=3.0$. The symbols (circles, up triangles and diamonds respectively) are the corresponding MC simulation data ${ }^{18}$.

Figure 2: Pair-correlation function $g(r)$ in the short attraction range for density $\rho \sigma^{3}=0.2$ (a), and $\rho \sigma^{3}=0.8$ (b). In both panels, the solid, dashed and dotted lines correspond respectively to the IE results for $\lambda=1.05$ and $k_{B} T / \varepsilon=0.5, \lambda=1.10$ and $k_{B} T / \varepsilon=0.5$, and $\lambda=1.20$ and $k_{B} T / \varepsilon=0.7$. The symbols (circles, up triangles and diamonds respectively) are the corresponding MC simulation data ${ }^{18}$.

Figure 3: Liquid-vapour phase diagram of a SW fluid for long attraction range, namely $\lambda=2.3, \lambda=2.5, \lambda=2.8$, and $\lambda=3.0$ from the bottom to the top. The thick lines and dashed lines correspond to the results of the IE method respectively for the binodal and spinodal lines. The dash-dotted lines represent the curve $\rho_{m} \sigma^{3}=\left(\rho_{l} \sigma^{3}+\rho_{v} \sigma^{3}\right) / 2$, where $\rho_{l} \sigma^{3}$ and $\rho_{v} \sigma^{3}$ are the densities of the liquid and vapour branches of binodal line. The thin lines are the SCOZA results ${ }^{24}$, while the open circles, up triangles and squares correspond respectively to the simulation data of Patel et al. ${ }^{13}$, Orkoulas and Panagiotopoulos ${ }^{12}$, and Benavides et al. ${ }^{8}$.

Figure 4: Liquid-vapour phase diagram of a SW fluid for intermediate attraction range, namely $\lambda=1.5, \lambda=1.75$, and $\lambda=2.0$ from the bottom to the top. The thick lines and dashed lines correspond to the results of our IE method respectively for the binodal and spinodal lines. The dash-dotted lines represent the curve $\rho_{m} \sigma^{3}=\left(\rho_{l} \sigma^{3}+\rho_{v} \sigma^{3}\right) / 2$, where $\rho_{l} \sigma^{3}$ and $\rho_{v} \sigma^{3}$ are the densities of the liquid and vapour branches of binodal line. The thin lines are the SCOZA results ${ }^{24}$, while the open circles correspond to the simulation data of Patel et al. ${ }^{13}$

Figure 5: Liquid-vapour phase diagram of a SW fluid for intermediate and short attraction range, namely $\lambda=1.175, \lambda=1.20, \lambda=1.25$ and $\lambda=1.375$ from the bottom to the top. The thick lines and dashed lines correspond to the results of our IE method respectively for the binodal and spinodal lines. The dash-dotted lines represent the curve $\rho_{m} \sigma^{3}=\left(\rho_{l} \sigma^{3}+\rho_{v} \sigma^{3}\right) / 2$, where $\rho_{l} \sigma^{3}$ and $\rho_{v} \sigma^{3}$ are the densities of the liquid and vapour branches of binodal line. The thin lines are the SCOZA results ${ }^{24}$, while the open circles and open squares correspond respectively to the simulation data of Patel et al. ${ }^{13}$ and del Rio et al. ${ }^{14}$. 


\section{List of tables}

Table I: Comparison of the contact values $g\left(\sigma^{+}\right), g\left(\lambda^{+}\right)$and $g\left(\lambda^{-}\right)$of the pair-correlation function obtained from our IE method with Monte Carlo data of ${ }^{18}$ for several values of the attraction range $\lambda$, density $\rho \sigma^{3}$ and temperatures $k_{B} T / \varepsilon$.

Table II: Coordinates of the critical point inferred from the integral equations method for different values of $\lambda$, along with the MC simulation data of Bolhuis et al. ${ }^{11}$, Reyes and Orea ${ }^{15}$ (a) and Orkoulas and Panagiotopoulos ${ }^{12}$ (b), as well as theoretical results from the SCOZA ${ }^{24}, \mathrm{HRT}^{23}$ and renormalization group $(\mathrm{RG})^{21}$ approaches. The error bars are $0.001 k_{\mathrm{B}} / \varepsilon$ for the temperature and $0.001 \sigma^{-3}$ for the density (see text). 
TABLE I

\begin{tabular}{|c|c|c|c|c|c|c|c|c|}
\hline \multirow[t]{2}{*}{$\lambda$} & \multirow[t]{2}{*}{$k_{B} T / \varepsilon$} & \multirow[t]{2}{*}{$\rho \sigma^{3}$} & \multicolumn{2}{|c|}{$g\left(\sigma^{+}\right)$} & \multicolumn{2}{|c|}{$g\left(\lambda^{-}\right)$} & \multicolumn{2}{|c|}{$g\left(\lambda^{+}\right)$} \\
\hline & & & IE & $\mathrm{MC}$ & IE & $\mathrm{MC}$ & IE & $\mathrm{MC}$ \\
\hline \multirow[t]{9}{*}{1.05} & 0.5 & 0.1 & 7.100 & 7.304 & 7.070 & 7.278 & 0.993 & 0.987 \\
\hline & & 0.5 & 5.178 & 7.662 & 4.990 & 7.374 & 1.32 & 0.999 \\
\hline & & 0.7 & 4.776 & 8.081 & 4.357 & 7.587 & 1.973 & 1.030 \\
\hline & 0.7 & 0.1 & 3.314 & 4.383 & 4.285 & 4.321 & 1.043 & 1.034 \\
\hline & & 0.5 & 4.651 & 5.557 & 4.423 & 2.237 & 1.421 & 1.257 \\
\hline & & 0.7 & 5.133 & 6.505 & 4.628 & 5.842 & 1.869 & 1.401 \\
\hline & 1.0 & 0.1 & 2.928 & 2.964 & 2.903 & 2.923 & 1.076 & 1.071 \\
\hline & & 0.5 & 3.885 & 4.336 & 3.645 & 4.009 & 1.562 & 1.477 \\
\hline & & 0.7 & 4.734 & 5.457 & 1.183 & 4.759 & 2.011 & 1.746 \\
\hline \multirow[t]{9}{*}{1.1} & 0.5 & 0.1 & 6.977 & 7.254 & 6.917 & 7.179 & 0.966 & 0.973 \\
\hline & & 0.5 & 4.350 & 6.088 & 4.134 & 5.816 & 0.93 & 0.787 \\
\hline & & 0.7 & 4.035 & 5.667 & 3.485 & 5.307 & 1.34 & 0.718 \\
\hline & 0.7 & 0.1 & 4.106 & 4.135 & 4.066 & 4.097 & 0.982 & 0.979 \\
\hline & & 0.5 & 3.892 & 4.334 & 3.608 & 4.028 & 1.060 & 0.966 \\
\hline & & 0.7 & 4.207 & 4.746 & 3.546 & 4.135 & 1.278 & 0.988 \\
\hline & 1.0 & 0.1 & 2.814 & 2.774 & 2.829 & 2.790 & 1.025 & 1.026 \\
\hline & & 0.5 & 3.354 & 3.565 & 3.006 & 3.186 & 1.237 & 1.172 \\
\hline & & 0.7 & 3.995 & 4.263 & 3.199 & 3.463 & 1.450 & 1.273 \\
\hline
\end{tabular}




\begin{tabular}{|c|c|c|c|c|c|c|c|c|}
\hline \multirow[t]{2}{*}{$\lambda$} & \multirow[t]{2}{*}{$k_{B} T / \varepsilon$} & \multirow[t]{2}{*}{$\rho \sigma^{3}$} & \multicolumn{2}{|c|}{$g\left(\sigma^{+}\right)$} & \multicolumn{2}{|c|}{$g\left(\lambda^{-}\right)$} & \multicolumn{2}{|c|}{$g\left(\lambda^{+}\right)$} \\
\hline & & & IE & $\mathrm{MC}$ & IE & $\mathrm{MC}$ & IE & $\mathrm{MC}$ \\
\hline \multirow[t]{9}{*}{1.2} & 0.7 & 0.1 & 4.093 & 4.126 & 4.002 & 4.030 & 0.965 & 0.968 \\
\hline & & 0.5 & 3.151 & 3.449 & 2.844 & 3.122 & 0.693 & 0.748 \\
\hline & & 0.7 & 3.308 & 3.281 & 2.579 & 2.810 & 0.708 & 0.675 \\
\hline & 1.0 & 0.1 & 2.679 & 2.671 & 2.624 & 2.628 & 0.965 & 0.968 \\
\hline & & 0.5 & 2.773 & 2.799 & 2.346 & 2.415 & 0.890 & 0.888 \\
\hline & & 0.7 & 3.284 & 3.173 & 2.269 & 2.372 & 0.901 & 0.871 \\
\hline & 1.5 & 0.1 & 2.009 & 2.016 & 1.953 & 1.956 & 1.003 & 1.004 \\
\hline & & 0.5 & 2.539 & 2.541 & 2.009 & 2.042 & 1.062 & 1.050 \\
\hline & & 0.7 & 3.253 & 3.168 & 2.010 & 2.063 & 1.087 & 1.059 \\
\hline \multirow[t]{9}{*}{1.5} & 1.5 & 0.1 & 1.955 & 1.952 & 1.836 & 1.832 & 0.941 & 0.941 \\
\hline & & 0.5 & 2.174 & 1.989 & 1.378 & 1.382 & 0.648 & 0.709 \\
\hline & & 0.7 & 3.069 & 2.783 & 1.191 & 1.115 & 0.454 & 0.590 \\
\hline & 2.0 & 0.1 & 1.661 & 1.661 & 1.563 & 1.563 & 0.944 & 0.945 \\
\hline & & 0.5 & 2.167 & 2.006 & 1.261 & 1.274 & 0.732 & 0.771 \\
\hline & & 0.7 & 3.150 & 2.880 & 1.063 & 1.063 & 0.563 & 0.646 \\
\hline & 3.0 & 0.1 & 1.448 & 1.44 & 1.350 & 1.351 & 0.966 & 0.968 \\
\hline & & 0.5 & 2.154 & 2.046 & 1.160 & 1.171 & 0.813 & 0.839 \\
\hline & & 0.7 & 3.169 & 2.998 & 0.974 & 0.982 & 0.651 & 0.704 \\
\hline
\end{tabular}




\begin{tabular}{|c|c|c|c|c|c|c|c|c|}
\hline \multirow[t]{2}{*}{$\lambda$} & \multirow[t]{2}{*}{$k_{B} T / \varepsilon$} & \multirow[t]{2}{*}{$\rho \sigma^{3}$} & \multicolumn{2}{|c|}{$g\left(\sigma^{+}\right)$} & \multicolumn{2}{|c|}{$g\left(\lambda^{-}\right)$} & \multicolumn{2}{|c|}{$g\left(\lambda^{+}\right)$} \\
\hline & & & IE & $\mathrm{MC}$ & $\mathrm{IE}$ & $\mathrm{MC}$ & IE & $\mathrm{MC}$ \\
\hline \multirow[t]{9}{*}{1.8} & 2.0 & 0.1 & 1.844 & 1.839 & 1.606 & 1.599 & 0.975 & 0.974 \\
\hline & & 0.5 & 2.293 & 2.199 & 1.201 & 1.175 & 0.691 & 0.714 \\
\hline & & 0.7 & 3.348 & 3.487 & 1.184 & 1.141 & 0.671 & 0.693 \\
\hline & 3.0 & 0.1 & 1.458 & 1.449 & 1.318 & 1.320 & 0.944 & 0.942 \\
\hline & & 0.5 & 2.211 & 2.162 & 1.086 & 1.079 & 0.760 & 0.773 \\
\hline & & 0.7 & 3.271 & 3.392 & 1.067 & 1.043 & 0.741 & 0.747 \\
\hline & 5.0 & 0.1 & 1.299 & 1.297 & 1.172 & 1.169 & 0.958 & 0.956 \\
\hline & & 0.5 & 2.171 & 2.152 & 1.005 & 1.005 & 0.815 & 0.822 \\
\hline & & 0.7 & 3.216 & 3.318 & 0.982 & 0.970 & 0.794 & 0.794 \\
\hline \multirow[t]{6}{*}{3.0} & 3.0 & 0.1 & 1.563 & 1.564 & 1.352 & 1.346 & 0.969 & 0.968 \\
\hline & & 0.5 & 2.362 & 2.424 & 1.213 & 1.199 & 0.865 & 0.861 \\
\hline & & 0.7 & 3.398 & 3.669 & 1.331 & 1.322 & 0.964 & 0.949 \\
\hline & 5.0 & 0.1 & 1.320 & 1.325 & 1.178 & 1.178 & 0.960 & 0.960 \\
\hline & & 0.5 & 2.151 & 2.300 & 1.114 & 1.109 & 0.912 & 0.910 \\
\hline & & 0.7 & 3.290 & 3.471 & 1.228 & 1.225 & 1.015 & 1.005 \\
\hline
\end{tabular}




\begin{tabular}{ccccccccccc}
\hline \hline & \multicolumn{2}{c}{$\mathrm{IE}$} & \multicolumn{2}{c}{$\mathrm{MC}$} & \multicolumn{2}{c}{$\mathrm{SCOZA}$} & $\mathrm{HRT}$ & $\mathrm{RG}$ \\
\hline$\lambda$ & $k_{B} T_{c}^{*} / \varepsilon$ & $\rho_{c} \sigma^{3}$ & $k_{B} T_{c}^{*} / \varepsilon$ & $\rho_{c} \sigma^{3}$ & $k_{B} T_{c}^{*} / \varepsilon$ & $\rho_{c} \sigma^{3}$ & $k_{B} T_{c}^{*} / \varepsilon$ & $\rho_{c} \sigma^{3}$ & $k_{B} T_{c}^{*} / \varepsilon$ & $\rho_{c} \sigma^{3}$ \\
\hline 1.175 & 0.552 & 0.252 & & & & & 0.656 & 0.452 & & \\
1.20 & 0.642 & 0.276 & & & & & 0.713 & 0.456 & & \\
1.25 & 0.792 & 0.352 & $0.765^{(a)}$ & $0.390^{(a)}$ & 0.761 & 0.343 & 0.767 & 0.415 & & \\
1.375 & 1.006 & 0.308 & $1.00^{(a)}$ & $0.345^{(a)}$ & 0.978 & 0.291 & 1.043 & 0.358 & & \\
1.5 & 1.218 & 0.255 & 1.219 & 0.299 & 1.210 & 0.272 & 1.211 & 0.272 & 1.218 & 0.310 \\
1.75 & 1.836 & 0.246 & 1.811 & 0.284 & 1.809 & 0.249 & 1.979 & 0.241 & & \\
2 & 2.708 & 0.287 & 2.764 & 0.225 & 2.699 & 0.252 & 3.182 & 0.275 & & \\
2.3 & 4.424 & 0.281 & - & - & 4.366 & 0.278 & 4.326 & 0.275 & & \\
2.5 & 5.776 & 0.276 & - & - & 5.712 & 0.275 & 5.524 & 0.271 & & \\
2.8 & 8.034 & 0.256 & - & - & 8.069 & 0.262 & 8.024 & 0.266 & & \\
3 & 9.768 & 0.232 & $9.99^{(b)}$ & $0.26^{(b)}$ & 9.941 & 0.258 & 9.925 & 0.621 & 9.877 & 0.257 \\
\hline \hline
\end{tabular}


Figure 1
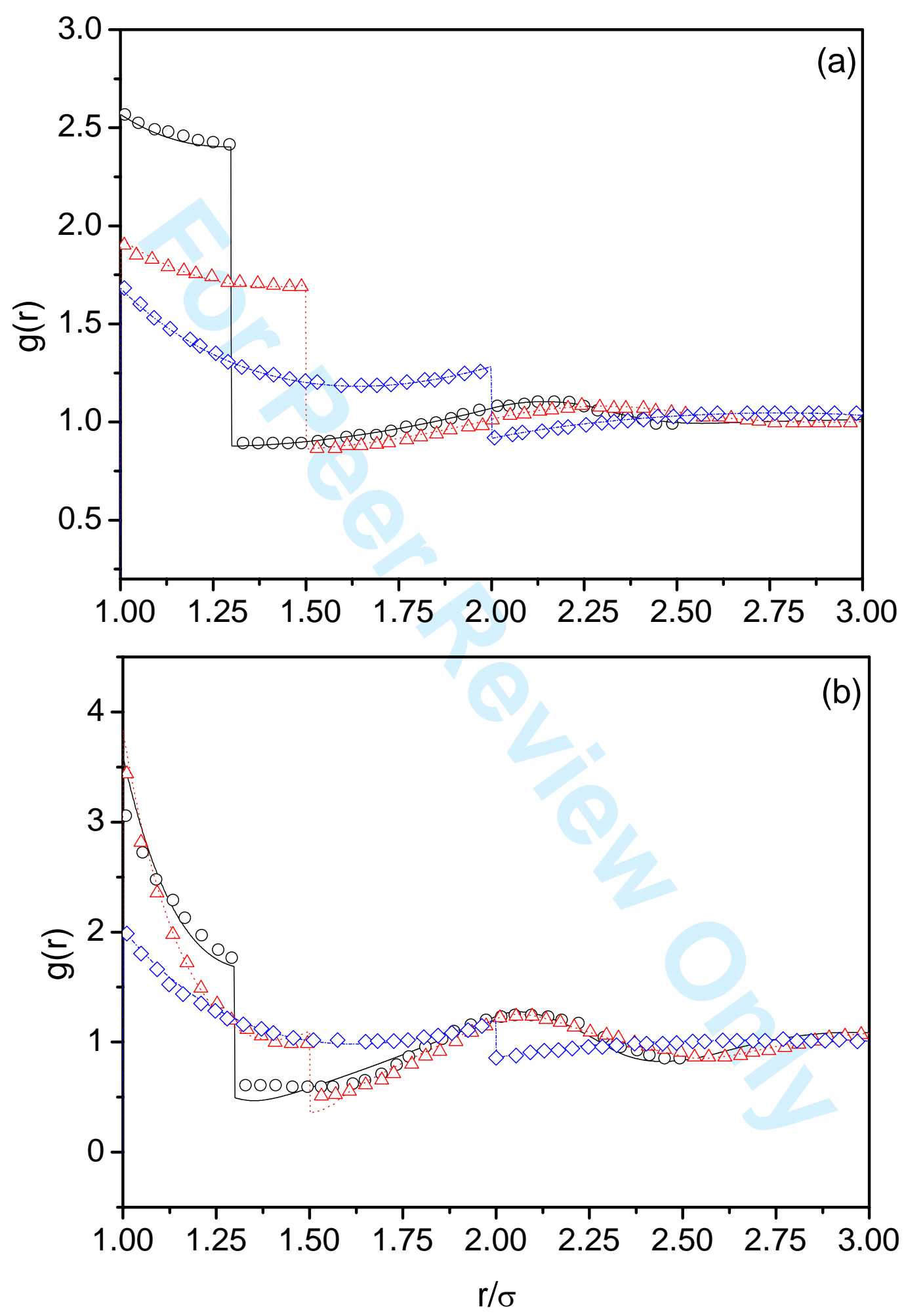
Figure 2
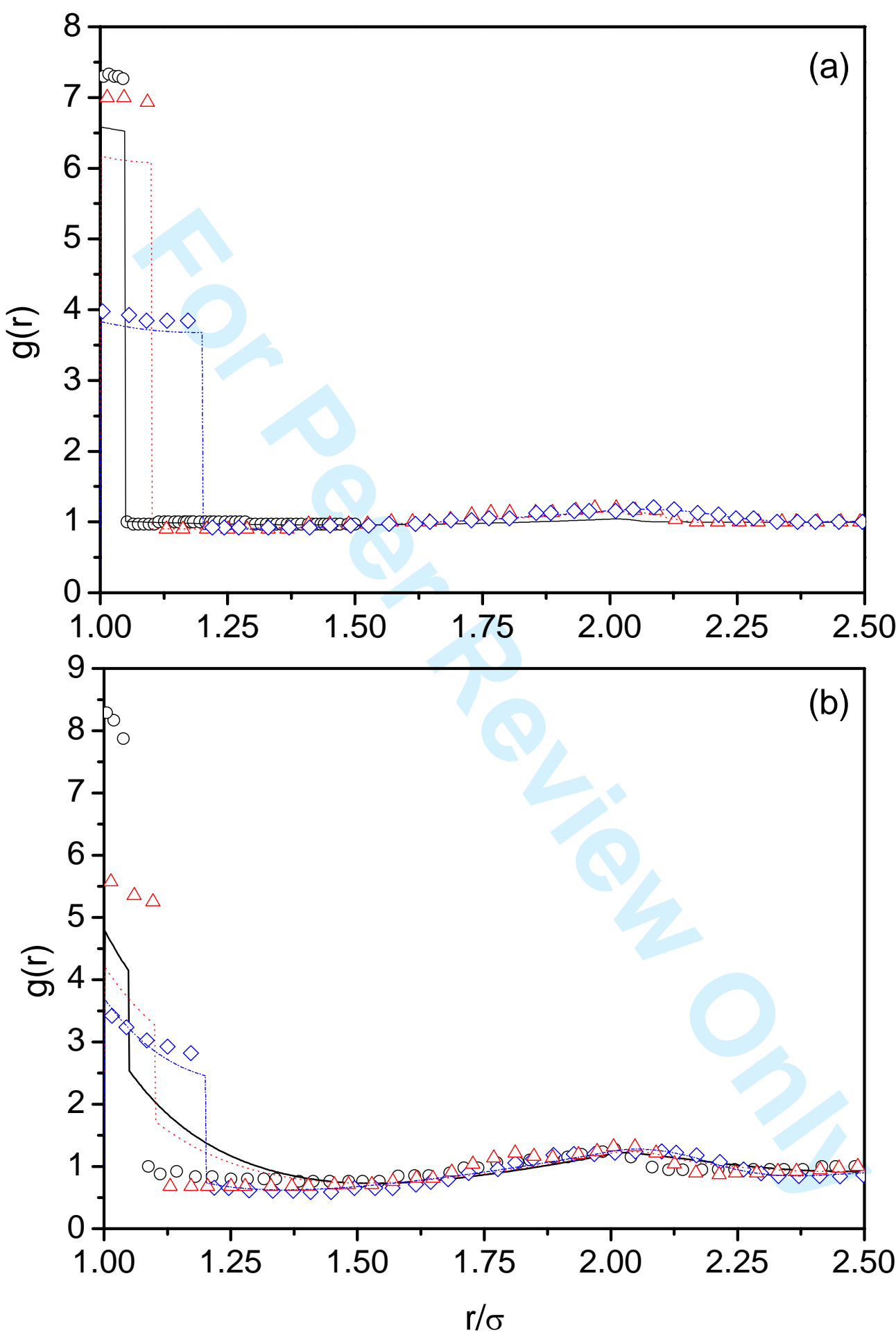
Figure 3

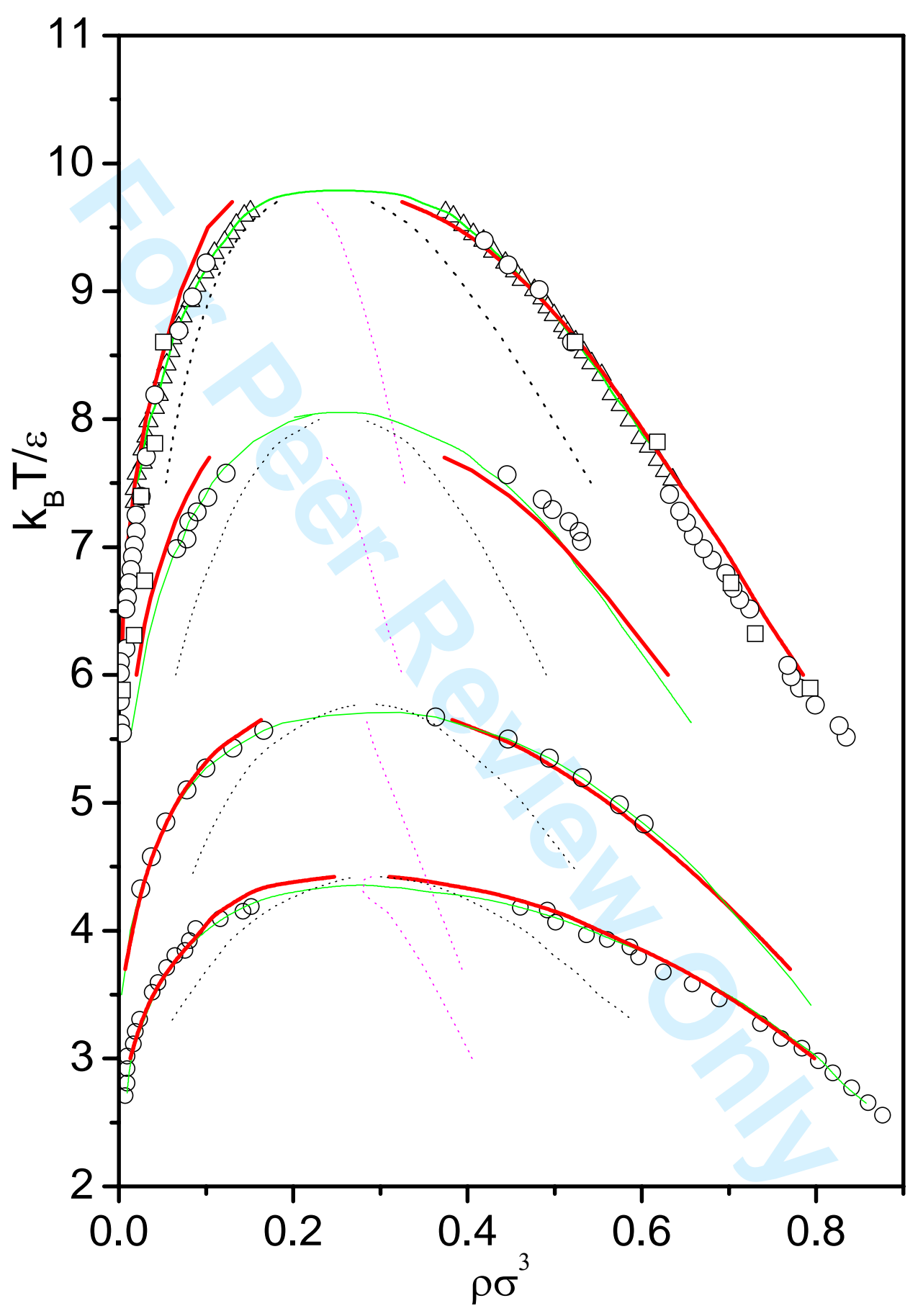


Figure 4

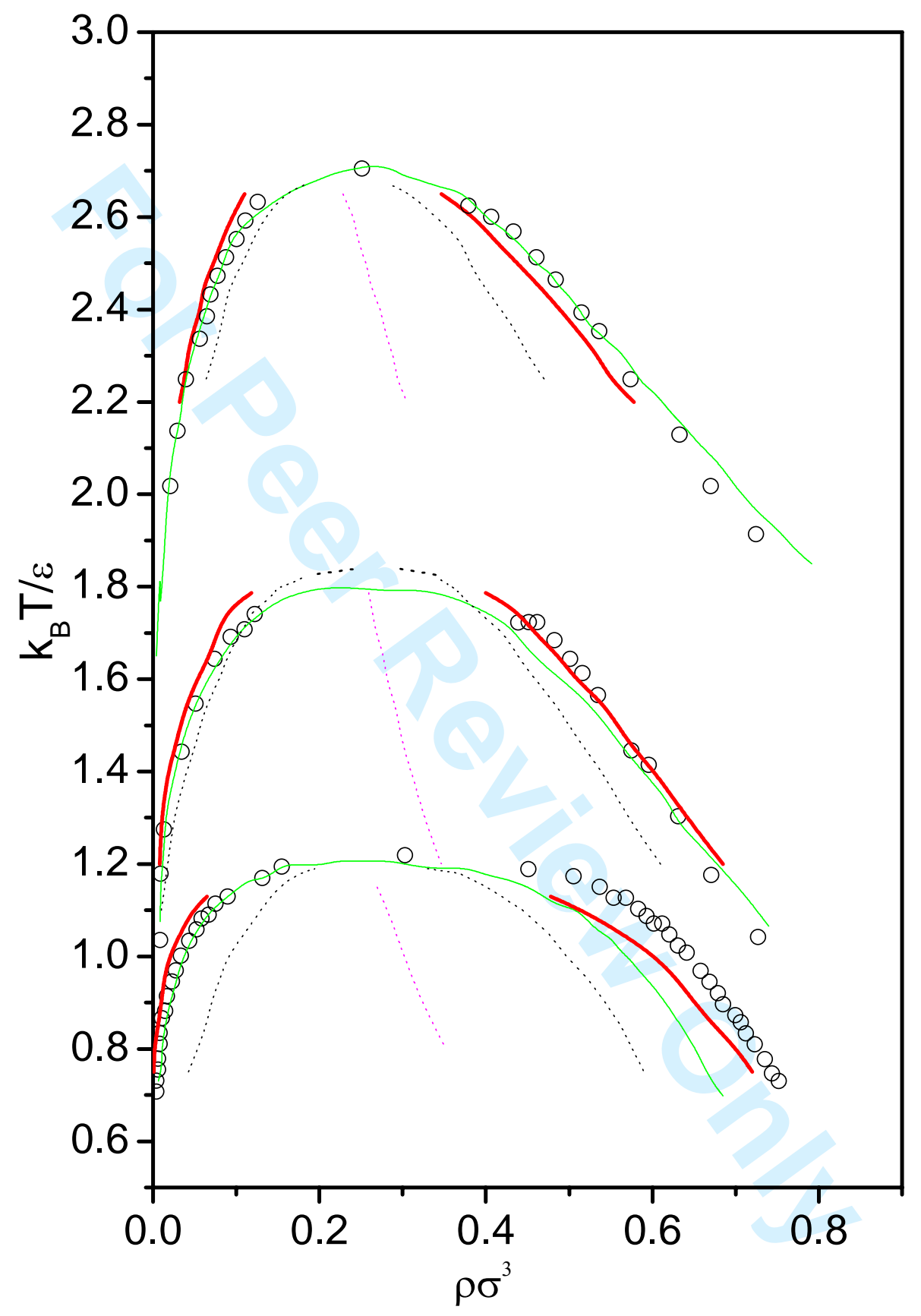


Figure 5

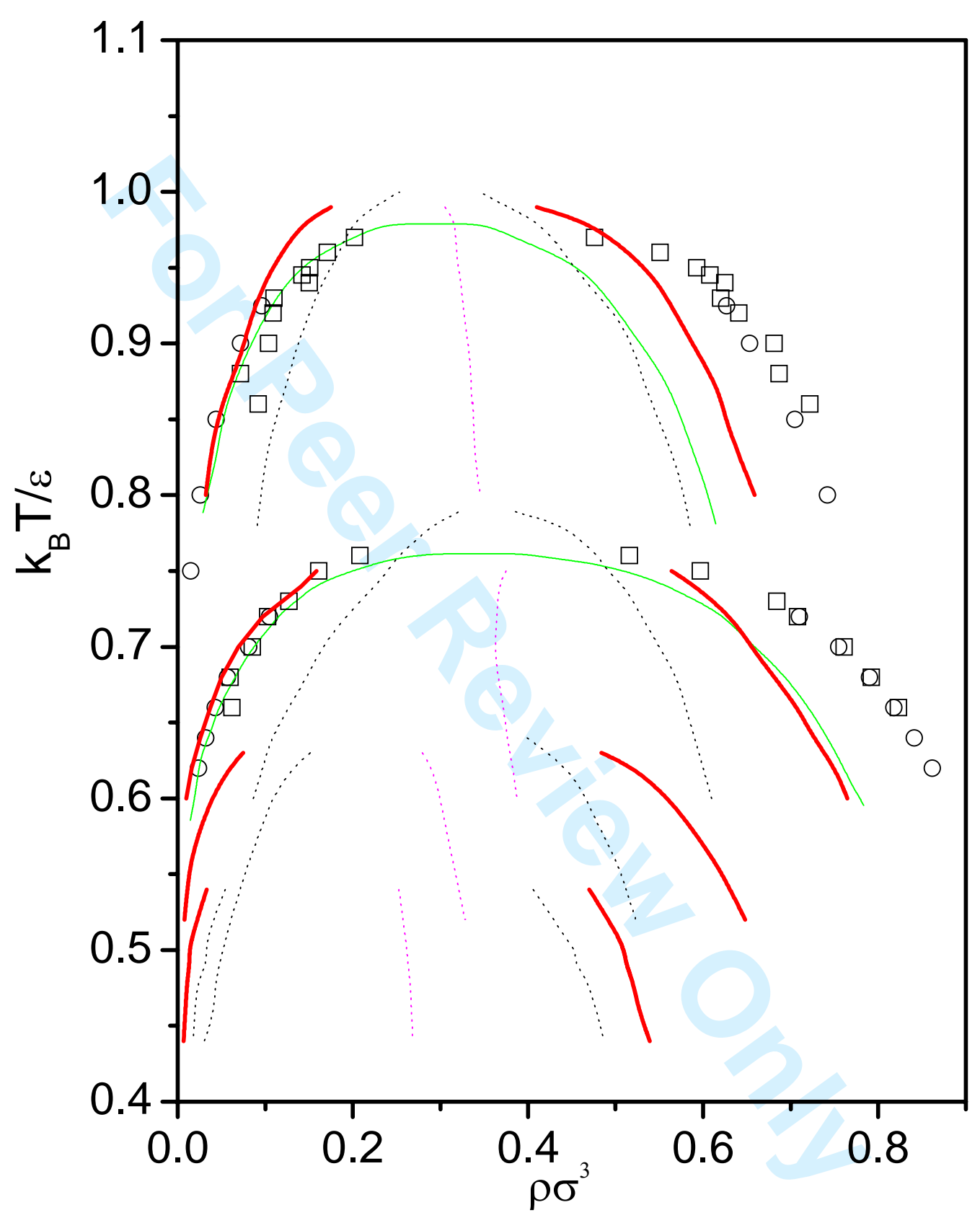



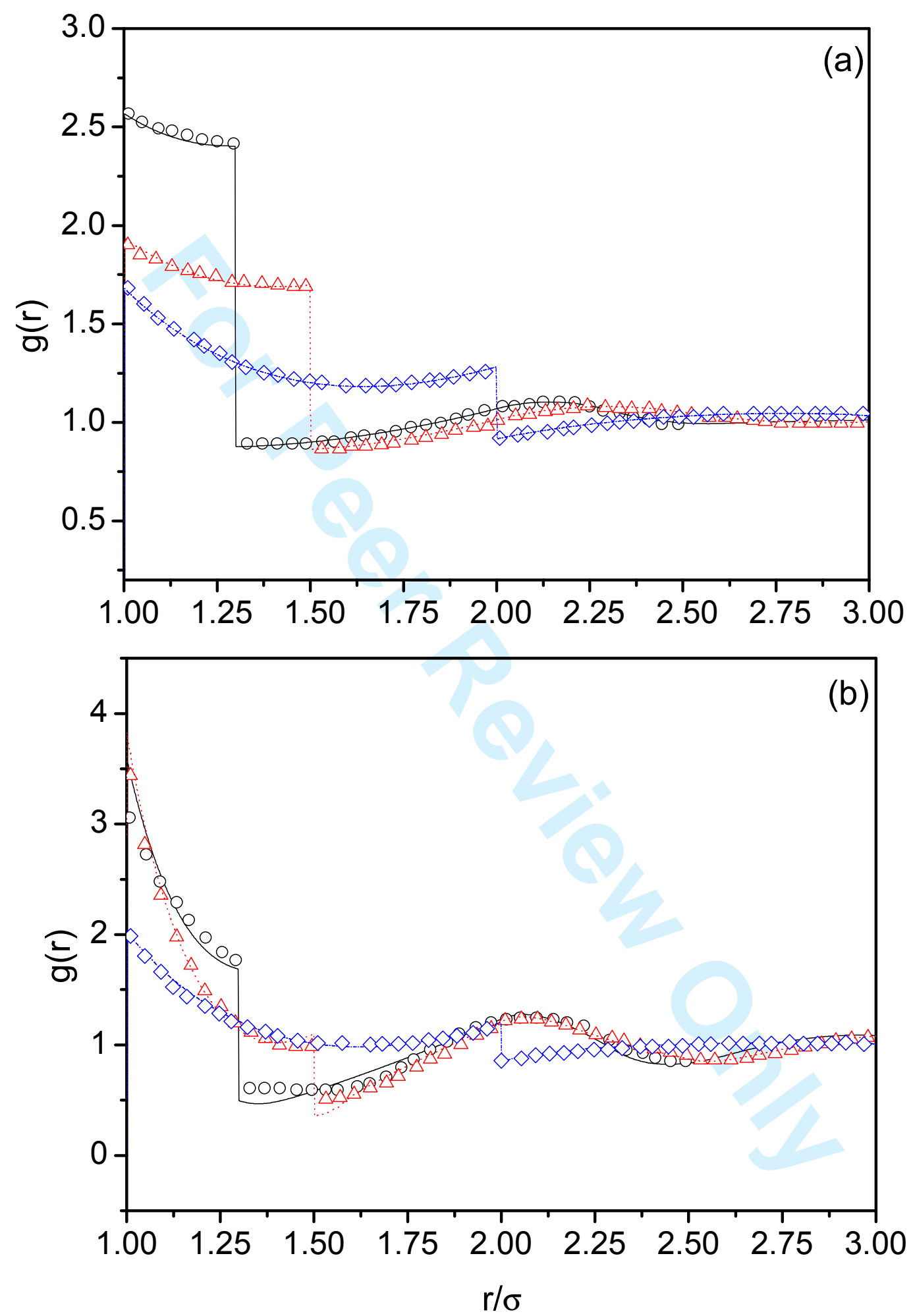

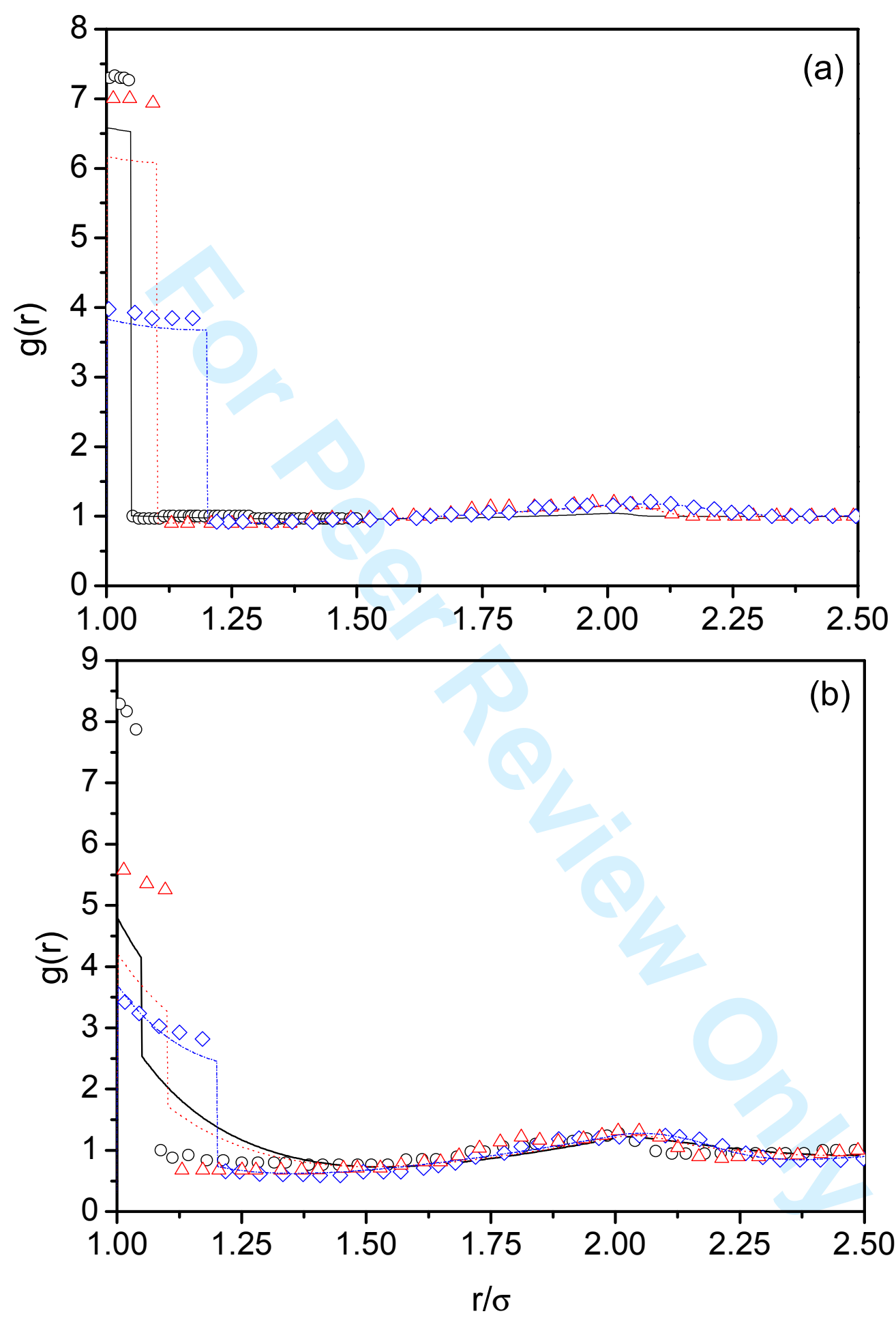


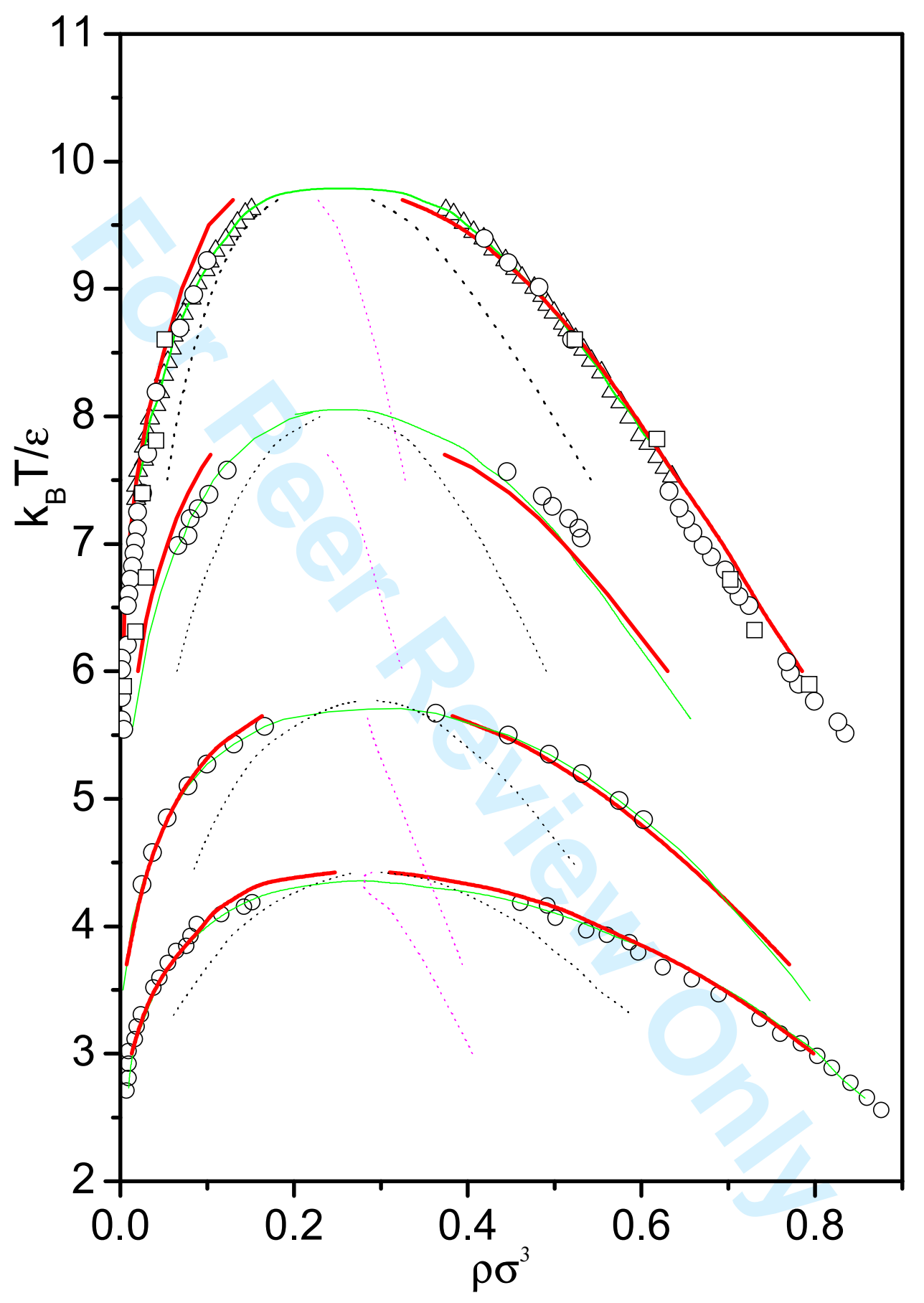




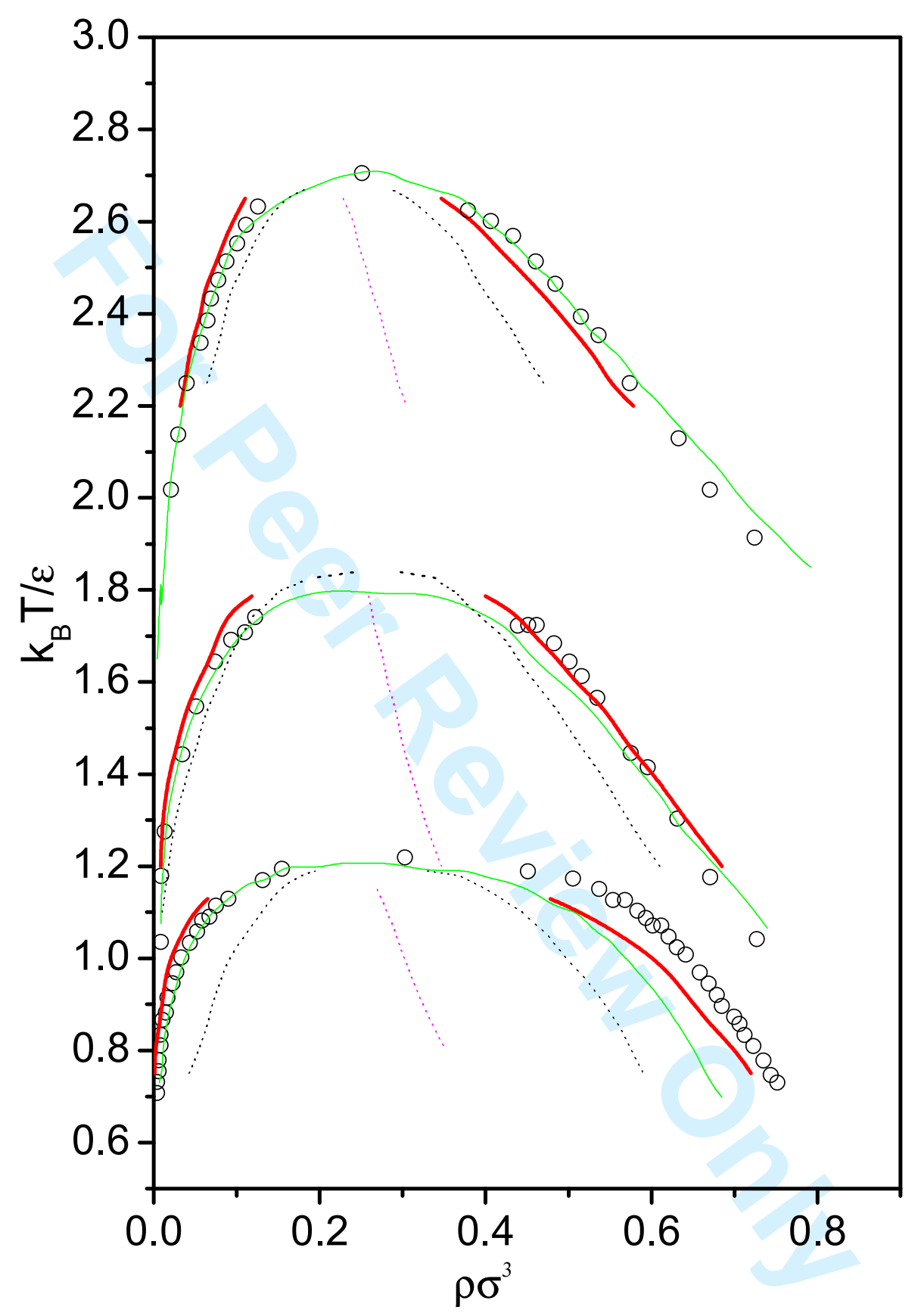




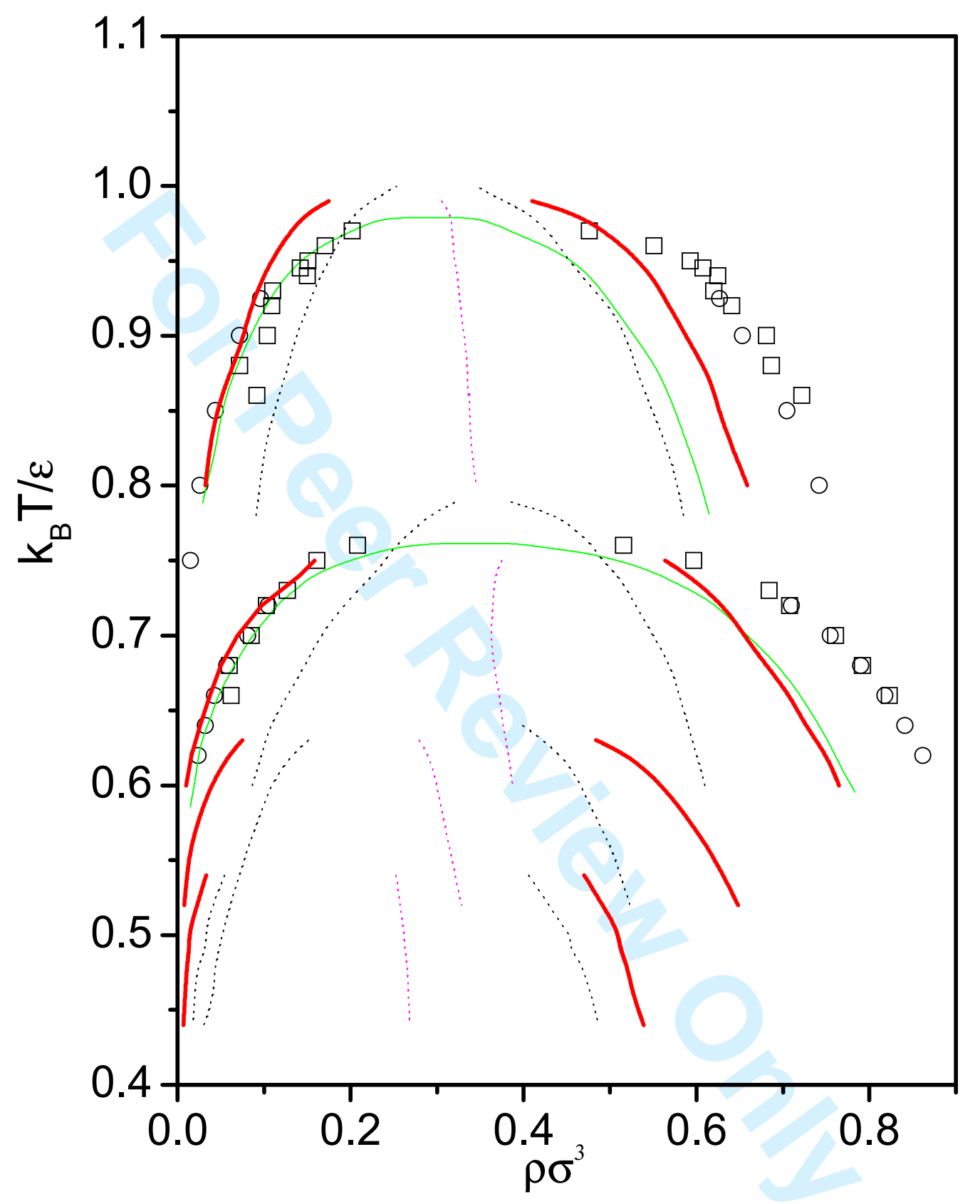

